\title{
Liver injury in remdesivir-treated COVID-19 patients
}

\author{
Rosa Zampino ${ }^{1,2} \cdot$ Ferruccio Mele $^{1} \cdot$ Letizia Lucia Florio $^{1} \cdot$ Lorenzo Bertolino $^{1} \cdot$ Roberto Andini $^{2}$ - Maria Galdo ${ }^{3}$. \\ Rosanna De Rosa ${ }^{4}$. Antonio Corcione ${ }^{4}$. Emanuele Durante-Mangoni ${ }^{1,2}$ (i)
}

Received: 27 May 2020 / Accepted: 16 July 2020 / Published online: 28 July 2020

(c) Asian Pacific Association for the Study of the Liver 2020

Novel Severe Acute Respiratory Syndrome coronavirus 2 (SARS-CoV-2) infection results predominantly in pulmonary involvement (Coronavirus disease 2019, COVID-19), but a direct, SARS-CoV-2-induced liver damage has also been described $[1,2]$. Thus, it is important to monitor liver function and evaluate hepatic safety of drugs administered to COVID-19 patients. Remdesivir (RDV), a nucleotide analogue RNA polymerase inhibitor, originally developed and tested for Ebola virus disease, showed in vitro efficacy against SARS-CoV-2 [3], and experience on its efficacy and safety for COVID-19 is accumulating $[4,5]$. However, hepatic safety of RDV in COVID-19 has not been the focus of detailed investigation. Here, we describe patterns of liver toxicity in 5 COVID-19 patients treated with RDV in the intensive care unit (ICU) of Monaldi Hospital, Naples, Italy, during March and April 2020. Overall, our Hospital cared for 32 critically ill COVID-19 patients.

Treatment was given in a compassionate use program (CPU) approved by our Ethics Committee. CPU was limited to the first 5 patients of our center who fulfilled all eligibility criteria (invasive mechanical ventilation, no multiorgan failure, no vasopressor requirement, ALT levels $<5$ xULN, creatinine clearance $>30 \mathrm{~mL} / \mathrm{min}$ ). RDV was administered intravenously as a $200 \mathrm{mg}$ loading dose, followed by $100 \mathrm{mg}$ daily over $1 \mathrm{~h}$ for up to 9 days. According to the early recommendations of the Italian Society for Infectious Diseases, Lombardy section, all patients had been previously treated

Emanuele Durante-Mangoni

emanuele.durante@unicampania.it

1 Internal Medicine, University of Campania 'L. Vanvitelli' and Units of, Napoli, Italy

2 Infectious and Transplant Medicine, AORN Ospedali dei Colli-Monaldi Hospital, Napoli, Italy

3 Pharmacy, AORN Ospedali dei Colli-Monaldi Hospital, Naples, Italy

4 Intensive Care, AORN Ospedali dei Colli-Monaldi Hospital, Naples, Italy with lopinavir/ritonavir (LPV/r, 400/100 mg twice daily po). Before and during RDV treatment, 4 of 5 patients also received hydroxychloroquine (HCQ, $200 \mathrm{mg}$ twice daily po). While on RDV, no patient received acetaminophen, patient 2 and 4 received ceftazidime-avibactam plus daptomycin and patient 3 meropenem and linezolid. None of the 5 treated patients had a previous history of liver disease, visceral obesity, viral hepatitis, or prior hepatotoxic medication or alcohol intake. Liver ultrasound did not show signs of advanced liver disease. Patient 1 and 2 had a history of hypertension and asthma, respectively, but were not receiving any relevant therapy in the ICU.

Figure 1 describes the dynamics of AST/ALT and bilirubin throughout the hospital stay, for each patient (Panels 1-5). In Panel 6, we report a comparison of median ALT and AST levels between RDV-treated patients and 5 COVID-19 patients who were treated in our Hospital ICU with the same schedule of LPV/r and HCQ, but without RDV. As shown in Panels 1-5, bilirubin increase occurred in 4 of 5 index patients on LPV/r. In contrast, the switch to RDV translated into a fast reduction of bilirubin and a significant increase in AST/ALT by day 3 of therapy in 4 of 5 patients. The single patient who did not receive HCQ with RDV (patient 4) did not show increase of ALT/AST levels. In no cases, RDV was discontinued because of liver injury. In patient 1, RDV was withdrawn at day 5 for a torsade de pointes requiring cardiac resuscitation, whereas patient 3 died on day 5 of RDV therapy. Final outcome was positive in $4 / 5$ patients.

Our observation supports previous findings obtained in healthy volunteers (Gilead Sciences, data on file) and COVID-19 patients treated with RDV [4, 5], suggesting this antiviral may cause hepatocellular injury. In our patients, this adverse effect neither progressed to severe liver damage nor induced liver failure, although none had a prior chronic liver disease. Although SARS-CoV-2 infection can cause aminotransferase elevation per se, 4 of our 5 patients had normal or slightly elevated AST/ALT levels at RDV treatment start, suggesting a direct role of RDV in hepatocellular toxicity. Despite the overall low number of patients 


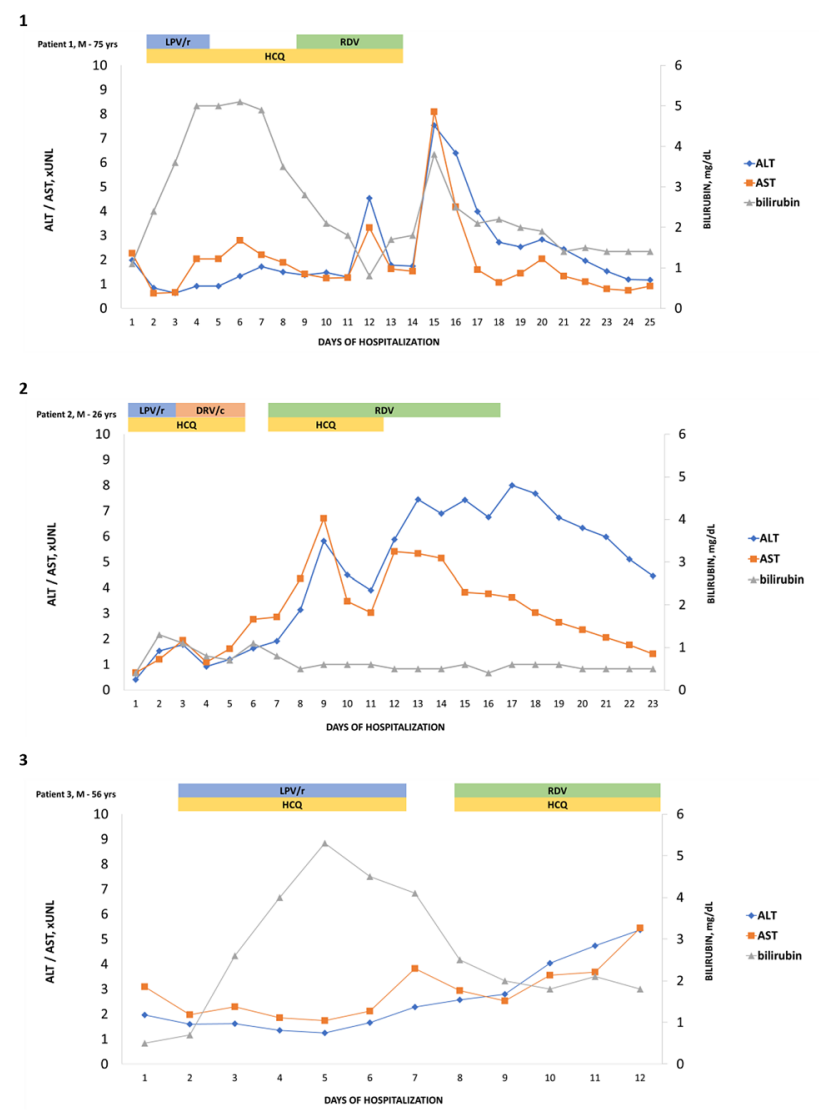

Fig. 1 Dynamics of ALT, AST and bilirubin in the 5 patients treated with Remdesivir (Panels 1-5). Aminotransferase values are shown as times the upper normal limit. Antivirals given are shown on top of each graph. Panel 6 shows a comparison of median ALT and AST levels between the 5 RDV-treated patients and 5 additional COVID-

treated, we observed a clear trend of bilirubin elevation with LPV/r and ALT/AST elevation with RDV. Our observation suggests RDV can be used with close monitoring of liver function tests and with caution in subjects with prior liver disease.

Acknowledgements Authors thank Gilead Sciences for providing Remdesivir for compassionate use.

Author contributions RZ, AC, EDM worked on concept of the study; FM, LLF, LB, RA, MG worked on data collection and data interpretation; RDR, RZ and EDM drafted the manuscript; all authors critically revised the manuscript.

Funding Remdesivir was provided by Gilead Sciences in a compassionate use program approved by EMA and our Institution Ethics Committee. No specific funding was received.

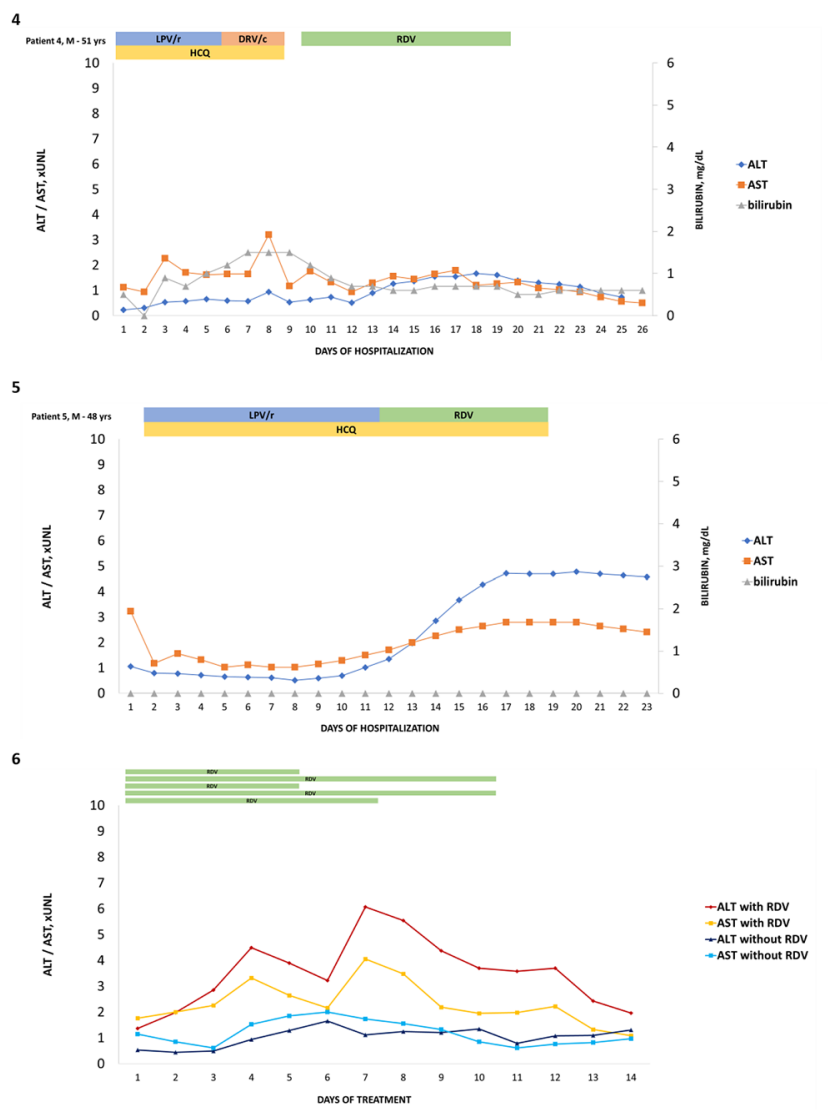

19 patients who were treated in our Hospital ICU with the same schedule of LPV/r and HCQ but without RDV. LPV/r lopinavir/ritonavir, $D R V / c$ darunavir/cobicistat, $H C Q$ hydroxychloroquine, $R D V$ remdesivir, $U N L$ upper normal limit

\section{Compliance with ethical standards}

Conflicts of interest The authors declared that they have no conflict of interest.

\section{References}

1. Zhao D, Yao F, Wang L, Zheng L, Gao Y, Ye J, et al. A comparative study on the clinical features of COVID-19 pneumonia to other pneumonias. Clin Infect Dis. 2020. https://doi.org/10.1093/ cid/ciaa247(pii:ciaa247).

2. Xu L, Liu J, Lu M, Yang D, Zheng X. Liver injury during highly pathogenic human coronavirus infections. Liver Int. 2020. https ://doi.org/10.1111/liv.14435.

3. Chan KW, Wong VT, Tang SCW. COVID-19: an update on the epidemiological, clinical, preventive and therapeutic evidence and guidelines of integrative Chinese-western medicine for the 
management of 2019 novel coronavirus disease. Am J Chin Med. 2020;13:1-26. https://doi.org/10.1142/S0192415X20500378.

4. Grein J, Ohmagari N, Shin D, Diaz G, Asperges E, Castagna A, et al. Compassionate use of remdesivir for patients with severe covid-19. N Engl J Med. 2020. https://doi.org/10.1056/NEJMo a2007016.

5. Wang Y, Zhang D, Du G, Du R, Zhao J, Jin Y, et al. Remdesivir in adults with severe COVID-19: a randomised, double-blind, placebo-controlled, multicentre trial. Lancet. 2020. https://doi. org/10.1016/S0140-6736(20)31022-9.

Publisher's Note Springer Nature remains neutral with regard to jurisdictional claims in published maps and institutional affiliations. 